\title{
Sample Preparation and Transfer Protocol for In-Vacuum Long-Wavelength Crystallography on Beamline I23 at Diamond Light Source
}

\author{
Ramona Duman ${ }^{1}$, Christian M. Orr ${ }^{1}$, Vitaliy Mykhaylyk ${ }^{1}$, Kamel El Omari ${ }^{1}$, Robert Pocock ${ }^{1}$, Vinay Grama $^{1}$, Armin Wagner $^{1}$ \\ ${ }^{1}$ Diamond Light Source Ltd, Harwell Science \& Innovation Campus
}

\section{Corresponding Author}

Armin Wagner

armin.wagner@diamond.ac.uk

\section{Citation}

Duman, R., Orr, C.M., Mykhaylyk, V., El Omari, K., Pocock, R., Grama, V., Wagner, A. Sample Preparation and Transfer Protocol for In-Vacuum Long-Wavelength Crystallography on Beamline I23 at Diamond Light Source. J. Vis. Exp. (170), e62364, doi:10.3791/62364 (2021).

\section{Date Published}

April 23, 2021

DOI

$10.3791 / 62364$

URL

jove.com/video/62364

\section{Abstract}

Long-wavelength macromolecular crystallography (MX) exploits the anomalous scattering properties of elements, such as sulfur, phosphorus, potassium, chlorine, or calcium, that are often natively present in macromolecules. This enables the direct structure solution of proteins and nucleic acids via experimental phasing without the need of additional labelling. To eliminate the significant air absorption of X-rays in this wavelength regime, these experiments are performed in a vacuum environment. Beamline I23 at Diamond Light Source, UK, is the first synchrotron instrument of its kind, designed and optimized for $\mathrm{MX}$ experiments in the long wavelength range towards $5 \AA$.

To make this possible, a large vacuum vessel encloses all endstation components of the sample environment. The necessity to maintain samples at cryogenic temperatures during storage and data collection in vacuum requires the use of thermally conductive sample holders. This facilitates efficient heat removal to ensure sample cooling to approximately $50 \mathrm{~K}$. The current protocol describes the procedures used for sample preparation and transfer of samples into vacuum on beamline 123. Ensuring uniformity in practices and methods already established within the macromolecular crystallography community, sample cooling to liquid nitrogen temperature can be performed in any laboratory setting equipped with standard $\mathrm{MX}$ tools.

Cryogenic storage and transport of samples only require standard commercially available equipment. Specialized equipment is required for the transfer of cryogenically cooled crystals from liquid nitrogen into the vacuum endstation. Bespoke sample handling tools and a dedicated Cryogenic Transfer System (CTS) have been developed in house. Diffraction data collected on samples prepared using this protocol show excellent merging statistics, indicating that the quality of samples is 
unaltered during the procedure. This opens unique opportunities for in-vacuum $M X$ in a wavelength range beyond standard synchrotron beamlines.

\section{Introduction}

Long-wavelength X-ray diffraction is used to harness the anomalous scattering properties of specific light atoms natively present in macromolecules. This helps to solve the crystallographic phase problem and to unambiguously confirm the identity and location of such elements within macromolecules. While in the early days of macromolecular crystallography, de novo structures were solved by multiple isomorphous replacement ${ }^{1}$, with the advent of tunable X-ray beamlines at synchrotrons, experimental phasing based on multi-wavelength and single-wavelength (SAD) anomalous diffraction techniques have become the dominant methods ${ }^{2}$. Both methods have historically relied on the isomorphous or anomalous signal from heavy metals, which need to be artificially introduced into the crystals by co-crystallization or crystal soaking ${ }^{3}$. The trial-and-error approach and unpredictable outcome can make these experiments frustratingly time-consuming. The incorporation of seleno-methionine during protein expression ${ }^{4}$ is a very elegant way to overcome these limitations and exploit anomalous diffraction at short wavelengths, although it can be very challenging in eukaryotic protein expression systems.

Long-wavelength $M X$ is extremely appealing for structure determination by native SAD experiments ${ }^{5,6}$ due to the convenience of using crystals directly from a successful crystallization trial without further treatment. Additionally, access to the absorption edges of elements of high biological importance, such as calcium, potassium, chlorine, sulfur, and phosphorus, opens the opportunity to directly identify the positions of these elements in macromolecules ${ }^{7,8,9,10}$. At medium and low resolution, element assignment based on the $2 \mathrm{~F}_{\mathrm{O}}-\mathrm{F}_{\mathrm{C}}$ electron density and chemical environment can be difficult, particularly for elements with similar number of electrons or weakly bound ions with partial occupancies. These ambiguities can be resolved by collecting data below and above the absorption edge of the element of interest and interpretation of the resulting model-phased anomalous difference Fourier maps ${ }^{11,12}$. Locating sulfur atom positions in these maps can also aid model-building into low-resolution electron density maps ${ }^{13}$. The absorption edges of these light elements are observed at wavelengths between $\lambda=3$ and 6 $\AA$ (see Figure 1, top). This wavelength range has been well beyond the capabilities of any synchrotron MX beamline, and efficient operation in this range requires overcoming several technical challenges, as outlined below.

Beamline I23 at Diamond Light Source, UK, is a unique instrument, specifically designed to facilitate long-wavelength $M X$ experiments, tunable in a wavelength range between $\lambda=$ 1.13 and $5.9 \AA$ (energy range between $E=2.1$ and $11 \mathrm{keV}$ ). By operating in a high-vacuum environment ${ }^{14}$, air absorption and scattering are eliminated, consequently enhancing the efficiency of diffraction experiments and the signal-to-noise ratio. A large vacuum endstation encloses all the components of the sample environment, including the semi-cylindrical Pilatus $12 \mathrm{M}$ detector, a multi-axis goniometer, the on-line viewing and collimation systems, as well as the bespoke equipment for sample transfer and storage (Figure 2). Each piece of equipment has been optimized to ensure that the best-quality long-wavelength data can be collected. The 
curved Pilatus 12M detector can collect to diffraction angles of $2 \theta= \pm 100^{\circ}$, resulting in sufficiently high-resolution diffraction data even at longest wavelengths (Figure 1, bottom). The 120 detector modules have been specifically selected for lowenergy compatibility and calibrations for an additional ultrahigh gain mode have been provided.

The lowest possible detector threshold is $1.8 \mathrm{keV}$, leading to increased corner and edge effects for energies lower than $3.6 \mathrm{keV}$ and compromised data quality at the longest wavelengths, particularly for low mosaicity crystals, can be observed. This effect in combination with the decrease in the detector quantum efficiency ${ }^{15}$ needs to be taken into consideration when planning an experiment. The multi-axis goniometer enables reorientation of crystals to allow for data collection strategies that maximize the quality and strength of the anomalous signal, as well as the completeness of the anomalous data collected. Sample absorption is a limiting factor for the experiments, particularly at longest wavelengths. Absorption corrections, as implemented in commonly used MX processing software packages ${ }^{16,17}$, are working well to wavelengths around $3 \AA$. Longer wavelengths will require analytical absorption corrections based on tomographic reconstructions ${ }^{18}$ or laser ablation to remove non-diffracting material and cut the crystals into well-defined shapes ${ }^{19}$. The latter will also assist in reducing the size of larger crystals as $\mathrm{X}$-ray diffraction experiments at longer wavelengths are more efficient for smaller crystals ${ }^{14}$. The challenge of keeping samples at cryogenic temperatures during data collection is addressed by conductive cooling, as using open-flow cold gas stream devices is not compatible with a vacuum environment. Hence, thermally conductive materials, such as copper, are needed for connecting the sample to a pulse tube cryocooler. The stainless steel SPINE standard pins used throughout MX, as well as any other commercially available sample mounts, are not suitable for invacuum long-wavelength MX because of their poor thermal conductivity.

The sample holders (SHs) for in-vacuum MX must be an essential part of the heat removal thermal pathway (Figure 3A). As such, they consist of a thermally conductive copper body and pin and include two important features: a strong magnet base to ensure an adequate thermal link to the cold goniometer head, and a sample mount, made from polyimide, to minimize X-ray absorption and scattering ${ }^{20}$. Efforts were made to ensure that the user experience of crystal-harvesting and flash-cooling is almost identical to that associated with standard MX practices. As the dedicated $123 \mathrm{SHs}$ are not directly compatible with other synchrotron beamlines, a stainless steel adaptor is used for compatibility with the crystal-harvesting magnetic wands and existing goniometer interfaces on other MX beamlines (Figure 3B). The adaptor is also important for making use of the automation facilities on other Diamond MX beamlines, which are based on ALStype robot gripper heads ${ }^{21}$ and unipuck-style base layouts ${ }^{22}$, if sample variation requires fast pre-screening for selection of the best diffracting crystals. The sample preparation and loading protocol can be broken down into two stages:

\section{Stage 1: Harvesting crystals and flash-freezing performed by users in their own laboratories}

Following assessment of the project suitability for 123 data collection, sample holders with loops matching the crystal sizes (pre-assembled with adaptors) are sent to user labs for crystal harvesting. To prevent any damage, $\mathrm{SH}$ and adaptors should not be separated and are to be used as one unit for the purpose of fishing crystals with appropriately sized loops using standard crystal-harvesting magnetic wands. As is common in MX, this task is performed manually under 
the microscope, and crystals are immediately flash-cooled in a foam dewar with liquid nitrogen ${ }^{23}$. Due to a mismatch of magnetic forces, the $\mathrm{SHs}$ are currently not compatible with unipucks. Storage and shipping are realized using combipucks (see the Table of Materials), which are available to users upon request, along with the compatible dry shipper inserts (Figure 3C). These pucks share the same base plate with the widely used unipucks and allow fast pre-screening of samples at other Diamond MX beamlines. Loaning this equipment to users is currently the best arrangement, until the bespoke sample holders are commercially available. Transport to the beamline requires the standard dry shippers used in the MX community.

\section{Stage 2: Transfer of cryo-cooled samples into the vacuum endstation}

Once the samples arrive on the beamline, they are prepared for transfer into the vacuum endstation. This involves removal of SHs from combipucks and separation from adaptors. Introducing biological samples to vacuum is routinely performed in the field of cryo-electron microscopy. Some of the well-established concepts were adapted for the I23 sample transfer. In short, SHs are transferred under liquid nitrogen onto transfer blocks (Figure 3D). These blocks have excellent thermal conductivity and a significant thermal mass, preventing the crystals from reaching the glass-transition temperature when in vacuum. Up to four blocks, with a capacity of four samples each, are loaded under liquid nitrogen into a block puck (Figure $\mathbf{3 H}$ ), which is used either for transferring samples to the Cryogenic Transfer System (CTS) or for storage in liquid nitrogen dewars between experiments.

The Cryogenic Transfer System developed at Diamond Light Source comprises of two sub-assemblies, the Sample Station and the Shuttle (Figure 4A). The Sample Station consists of a liquid nitrogen bath for temporary storage of protein crystals and has specific features to ensure safety and allow a userfriendly experience (Figure 5). The CTS is controlled by a programmable logic controller via a user-friendly touchscreen interface. The Sample Station has light-emitting diodes built in for better visualization and a set of heaters controlled in close-loop to automate the drying of the liquid nitrogen bath once the samples have been transferred. It also has a variety of sensors to ensure safety and efficient functioning of the system. The Sample Station has bespoke hardware to provide a reliable electrical interface to interact with the shuttle for operations, such as pumping down to rough vacuum for sample transfer, as well as monitoring of liquid nitrogen levels and the temperature inside the shuttle.

The Shuttle (Figure 6) is a portable device used to pick up a transfer block from the Sample Station liquid nitrogen bath and transfer it inside a cryogenic and vacuum environment to the endstation. It includes a liquid nitrogen dewar to keep the samples cold during transfer, liquid level monitoring in the dewar, and a variety of sensors for operation and user safety. The transfer arm is equipped with a magnetic drive and includes machined grooves to guide users in safely loading and unloading transfer blocks into the endstation. Transfer from the shuttle to the vacuum vessel is conducted via an airlock. The airlock is an interface for the shuttle on the endstation used to evacuate the interspace between the shuttle and the endstation, before opening the shuttle and endstation vacuum valves. The pumping and venting sequences are fully automated and can be operated via a large touchscreen with a user-friendly interface (Figure 4C). The current protocol is used to transfer a thaumatin crystal to the vacuum endstation for data collection. 


\section{Protocol}

\section{Crystal harvesting}

NOTE: Use appropriate personal protective equipment: goggles and gloves, where possible.

1. After the SHs arrive at the user lab in combipucks (Figure 3C), separate the lid from the base of the combipuck such that the SHs remain attached to the base, and vials are retained in the lid.

2. Immerse the lid with vials in liquid nitrogen. Attach an $\mathrm{SH}+$ adaptor (Figure 3B, right) to a magnetic wand, and harvest crystals as usual.

3. Flash-cool each sample directly into the combipuck, making note of the sample position. To close the puck, use a puck wand to attach the base to the lid.

4. Transfer the combipuck from liquid nitrogen to the dry shipper or liquid nitrogen storage dewar. Ship the dry shipper to Diamond (https://www.diamond.ac.uk/Instruments/Mx/ Common/Common-Manual/Shipping-Samples.html).

\section{Sample transfer to vacuum}

1. Loading of $\mathrm{SH}$ from combipuck to the transfer block

1. Place the base of the block puck (Figure $3 \mathbf{H}$ ) already populated with empty transfer blocks (Figure 3D) onto its support base inside the liquid nitrogen in a foam container (Figure 3J-b).

NOTE: The orientation of the transfer blocks is important for the accuracy of sample transfer inside the vacuum vessel. As such, the blocks should be placed onto the block puck base making sure the pin marked with an arrow in Figure 3D is on the left of the block.

2. Place the vial puck in the foam container filled with liquid nitrogen, making sure the base of the puck is secured to the magnetic holder inside the foam container (Figure $\mathbf{3} \mathbf{J}-\mathbf{a})$.

3. Pre-cool all necessary tools in liquid nitrogen. Use the puck separator tool shown in Figure $\mathbf{3 G}$ on the high setting $\mathbf{H}$ to separate the lid from base, such that the base remains attached to the magnetic holder and the SHs are exposed inside the liquid nitrogen.

4. To remove each $\mathrm{SH}$ from its adaptor, use the separator wand (Figure 3F) to pick up the $\mathrm{SH}$ from the combipuck base, and place into the appropriate position of the transfer block in the horizontal position of the carousel in Figure 3J-b.

1. Place the separator wand over the $\mathrm{SH}+$ adaptor as far down as it can go, making sure the wand is vertical, to avoid touching the sample.

2. Move the small lever on the separator wand down with the thumb until it clicks, to secure the $\mathrm{SH}$ inside and pull the $\mathrm{SH}$ from the adaptor.

3. Lower the separator over the desired block position, making sure one of the three prongs fits inside the central hole of the block.

4. Release the $\mathrm{SH}$ by moving the lever back up. Repeat these steps for each SH.

5. To load samples into the next sample block, use the carousel key tool (Figure 3E) to rotate an empty block into the horizontal position. 
6. Attach the puck separator tool shown in Figure $\mathbf{3 G}$ using the low setting $L$ to the lid of the block puck by screwing clockwise.

7. Once all SHs are transferred, to close the block puck, place the lid in liquid nitrogen and wait for the temperature to equilibrate, then fit the lid over the base as in Figure 3I. With the separator tool attached, lift gently to release from the carousel.

8. At this stage, the block puck can be transferred to the CTS (Figure 4B) or to a liquid nitrogen storage dewar.

2. Loading of transfer blocks into the vacuum vessel

1. Ensure the shuttle is securely attached to the station. Open the nitrogen gas and air valves, and ensure gases are flowing. Switch on the CTS.

2. If no warning messages are apparent on the display, proceed with cooling down both the bath and the shuttle with liquid nitrogen. Place the supplied funnel in the filling port on the shuttle, and slowly pour liquid nitrogen into the funnel whilst monitoring the level on the screen. Stop when the indicator turns from red to blue.

NOTE: The shuttle is ready to use when the temperature of the cold seat displayed on the touchscreen is below $100 \mathrm{~K}$. The Sample Station bath can be filled simultaneously using the correct funnel to the level marked on the wall of the bath or $100 \%$ on the liquid nitrogen level display. Liquid nitrogen levels and temperature sensors should be monitored constantly throughout operation; several top-ups will be required.

3. Once the shuttle cold seat temperature is below 100 $\mathrm{K}$ and liquid nitrogen levels on the shuttle and bath stabilize, transfer a block puck from liquid nitrogen to the CTS bath using the attached puck separator tool. Remove the lid of the block puck, and close the lid of the CTS bath.

4. To introduce a block into the shuttle, open the CTS valve, if not already open, by pressing the Open Shuttle Valve button on the display. Unlock the shuttle handle by rotating $90^{\circ}$ clockwise, and advance it towards the bath so that the guided track on the handle enforces the correct path of travel towards the bath. Once the block cover is visible inside the bath, allow the cover to cool. After the bubbling of liquid nitrogen around the cover has stopped, advance to the transfer block.

5. To lock the transfer block onto the shuttle, rotate the handle $180^{\circ}$ clockwise.

6. Retract the handle to the original back position, and then 'Lock' it in place by rotating $90^{\circ}$ anti-clockwise.

7. Press Close Shuttle Valve \& Pump on the display screen to start shuttle evacuation.

8. Once the message Shuttle ready to detach is displayed on the touchscreen, press the lever underneath the shuttle, and carefully lift it using the handle at the top.

9. Carry the shuttle to the airlock on the vacuum endstation in an upright position.

10. Attach the shuttle to the airlock on the vacuum endstation.

NOTE: Once securely attached, the touchscreen on the endstation will confirm the status of the shuttle and interlock. 
11. Select an empty block position within the vessel by pressing the corresponding button on the touchscreen and moving the sample hotel to the correct loading position.

12. Once the sample hotel is in position, the Open button will become active. Press this button to initiate the vacuum interlock sequence.

NOTE: The pump will start, and the progress will be displayed on the monitor. This may take up to two minutes to complete.

13. After the sequence is complete, the status will change to Airlock open, transfer in progress. Twist the handle $90^{\circ}$ clockwise to unlock the rod, and gently push the rod into the vessel so that the guided track again enforces the correct path of travel towards the sample hotel position. Using the video feed displayed on the screen for guidance, slowly insert the block into the hotel, ensuring that the block position light on the touch display is activated. Once activated, rotate the handle $180^{\circ}$ anti-clockwise to release the block, and pull the rod out of the vessel. Once fully retracted, rotate the handle $90^{\circ}$ anticlockwise to lock the rod.

14. Once the rod is locked, the Close button will become active. Press this to close the end station vacuum valve, and vent the space between the shuttle and the vessel to atmospheric pressure, waiting for up to $20 \mathrm{~s}$ for completion.

15. Wait for the display to show the status okay to remove shuttle once the sequence is completed. At this point, remove the shuttle, and return to the CTS to repeat the process for the next block.
16. To prepare the next block for transfer, rotate the block puck inside the bath. Push the built-in rotation key on the top of the acrylic lid down into the lock in the center of the block puck. Whilst holding it down, turn the key to position the desired block in the pickup position.

17. Once all blocks have been transferred, ensure the shuttle valve is open whilst it is mounted on the CTS. Press the bake button on the touchscreen, and select both bath and shuttle, then press bake.

NOTE: This warms up both the shuttle and bath to boil off the liquid nitrogen and subsequently evaporate any accumulated ice/condensation before the next use. Once the bake has started, the gas and air can be switched off.

\section{Representative Results}

A thaumatin crystal was introduced to the vacuum endstation using the protocol outlined above. Diffraction data were collected at a wavelength of $2.7552 \AA(E=4500 \mathrm{eV})$ as 3600 images with a rotation increment of $0.1^{\circ}$ and $0.1 \mathrm{~s}$ exposure per image. The beam size was adjusted to $150 \mu \mathrm{m} \times 150$ $\mu \mathrm{m}$ and reduced to $10 \%$ transmission, with a corresponding flux measurement of $7.1 \times 10^{9}$ photons/s. The choice of $\lambda=$ $2.7552 \AA$ is based on a compromise between the increase in anomalous signal and sample absorption effects and the decrease in resolution to longer wavelengths. Although not close to the theoretical absorption edge of sulfur $(\lambda=5.0095$ $\AA$ ), at this wavelength, the imaginary contribution to the scattering factor of sulfur $\mathrm{ft}^{\prime \prime}$ is $1.57 \mathrm{e}^{-}$, a factor of 1.6-2.1 larger compared to wavelengths between 1.7 and $2 \AA$. The resulting stronger anomalous signals allow successful S-SAD phasing for more challenging projects. 
A variety of difficult phasing experiments have already been carried out on beamline $123^{24,25,26,27}$, with data collected at this wavelength. While phasing by S-SAD is possible using much shorter wavelengths, this often requires building up anomalous signal through merging data from many isomorphous crystals to reach multiplicity values over $100^{28}$. Due to the enhanced anomalous signal at longer wavelengths, most phasing projects solved on 123 only required data from one crystal. A representative diffraction image is shown in Figure 7, left. Data processing using Xia2-3dii ${ }^{29}$ produced excellent merging statistics, as outlined in Table 1. Figure 7, right, shows part of a representative diffraction image from the thaumatin data set and illustrates the low background surrounding the Bragg reflections, which contributes to the large $I / \sigma(I)$ values typically observed in the vacuum setup, ensuring that only X-rays scattered by the sample reach the detector.
The maximum achievable resolution of $1.8 \AA$ is due to the detector geometry and the chosen wavelength of the Xray radiation. The dataset yielded very strong anomalous signal, reflected in the mid-slope of anomalous normal probability parameter of 2.677 , facilitating structure solution by the automatic phasing pipeline CRANK2. The high quality of the resulting electron density map enabled successful automatic model building by the Buccaneer ${ }^{30}$ module within CRANK2 ${ }^{31}$, with correct placement for $100 \%$ of the amino acid sequence of thaumatin. The phased anomalous difference Fourier map, calculated with $\mathrm{ANODE}^{11}$, reveals 16 very well-ordered sulfur atoms and one sulfur atom from Cys 159 with two alternative conformations, as confirmed by the 18 significant heights of the peaks at the positions of the anomalous scatterers in Table 2. The 16 cysteine residues within thaumatin form 8 disulfide bridges, which are all clearly visible in the 2Fo-Fc map (Figure 8). 

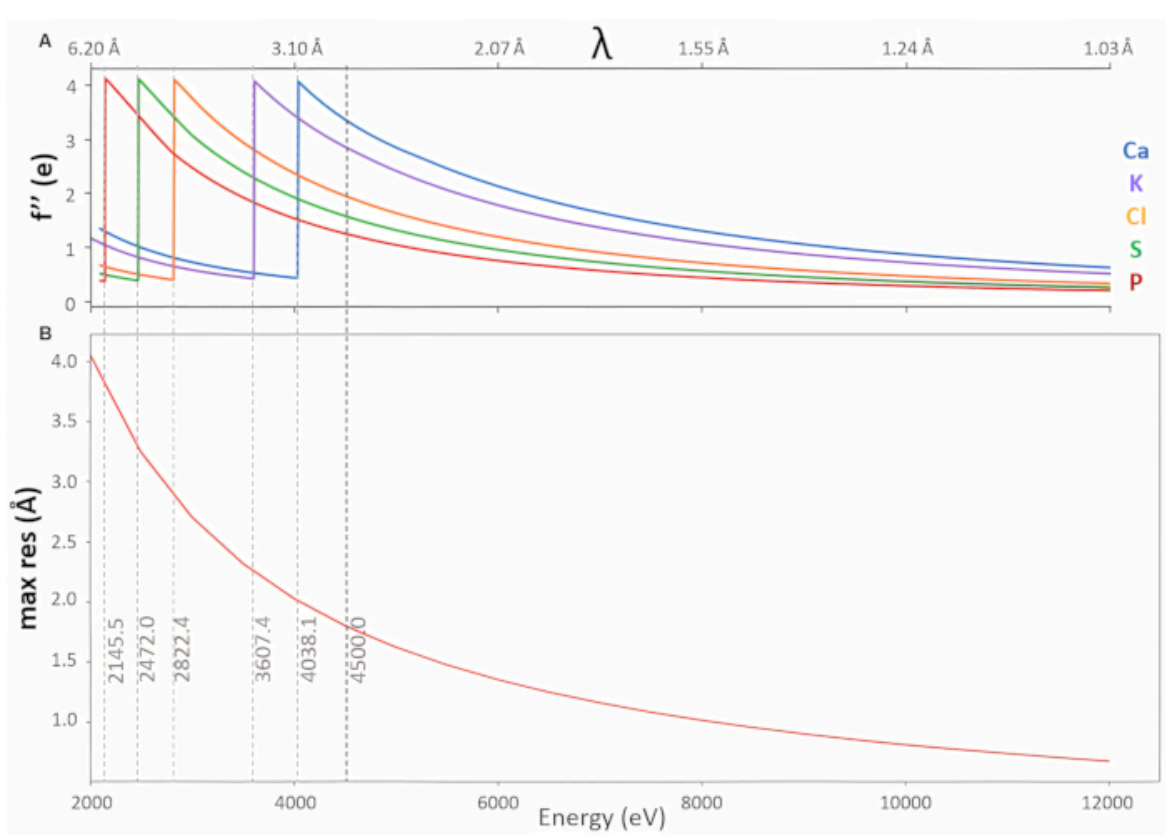

Figure 1: High-resolution diffraction data from long-wavelength MX experiments. (A) Plot of f" values against energy, indicating absorption edges of light elements accessible on beamline I23. (B) Maximum resolution achievable at the corners of the P12M detector against energy. Abbreviation: MX = macromolecular crystallography. Please click here to view a larger version of this figure. 


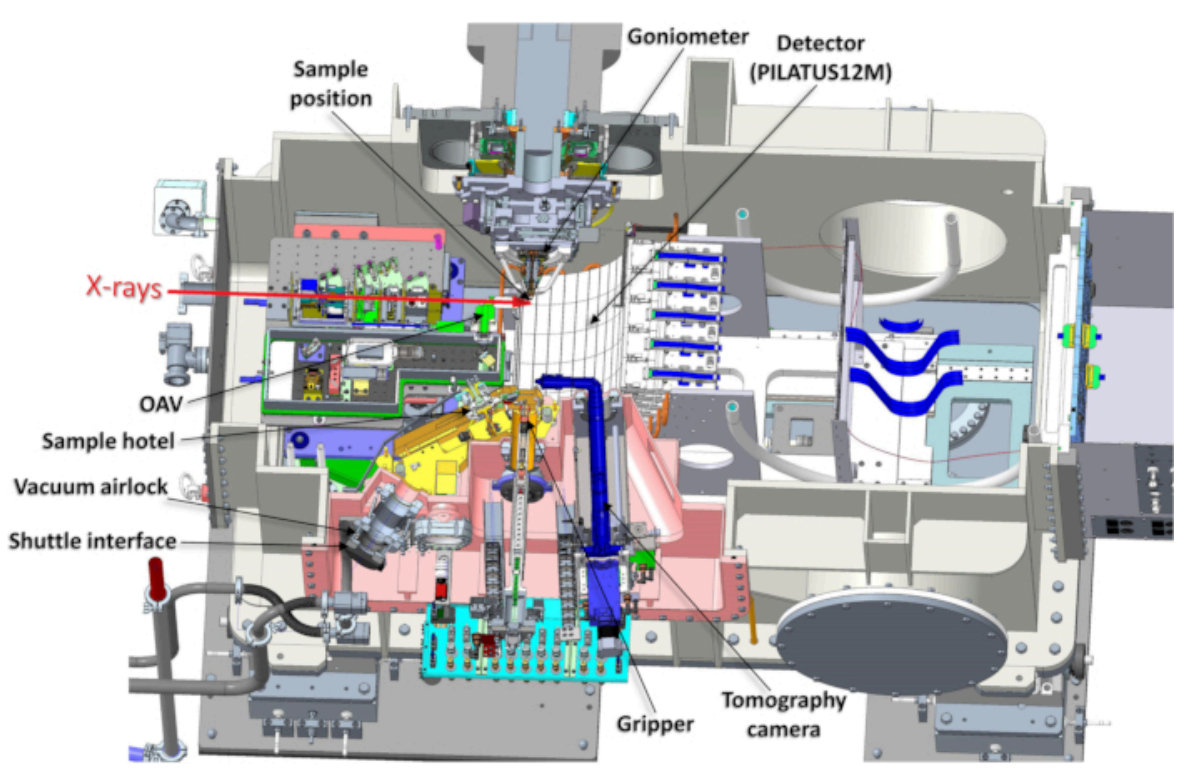

Figure 2: Horizontal section through the vacuum vessel with all the components of the endstation. Abbreviation: OAV = on-axis viewing system. Please click here to view a larger version of this figure. 


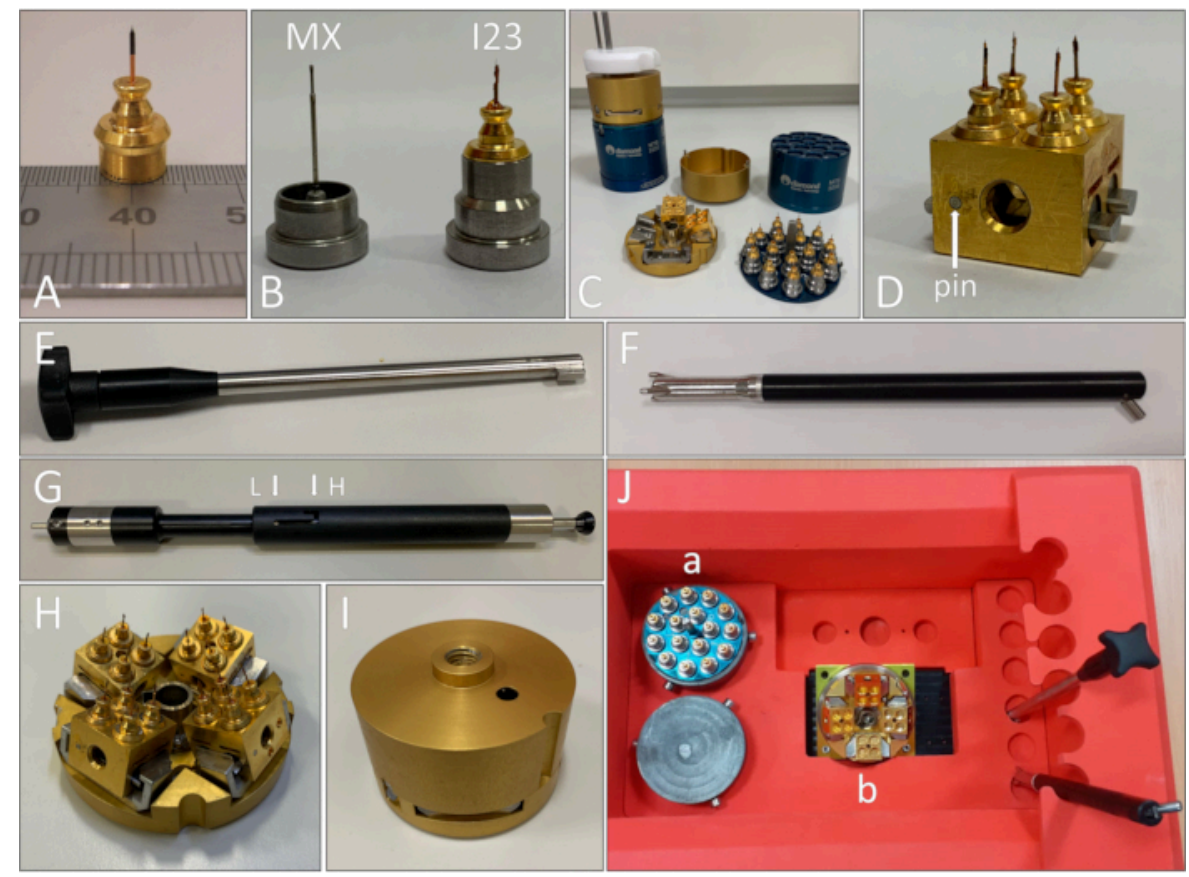

Figure 3: Sample handling tools. (A) I23 Sample holder. (B) MX spine-standard pin (left) next to an I23 sample holder with adaptor (right). (C) Combipuck lid and base with 123 sample holders (blue). Block puck lid and base with two transfer blocks (gold). A dry shipper cane, compatible with both combipucks and block pucks, is visible at the back. (D) Transfer block with four 123 sample holders. (E) Key tool used for rotation of the block puck base. (F) Separator wand. (G) Puck separator tool with two arrows showing the high and low settings. (H) Block puck base with four empty Cu blocks. (I) Lid for the block puck. (J) Foam container with all necessary tools for transferring sample holders from combipuck bases to copper blocks. Please click here to view a larger version of this figure. 

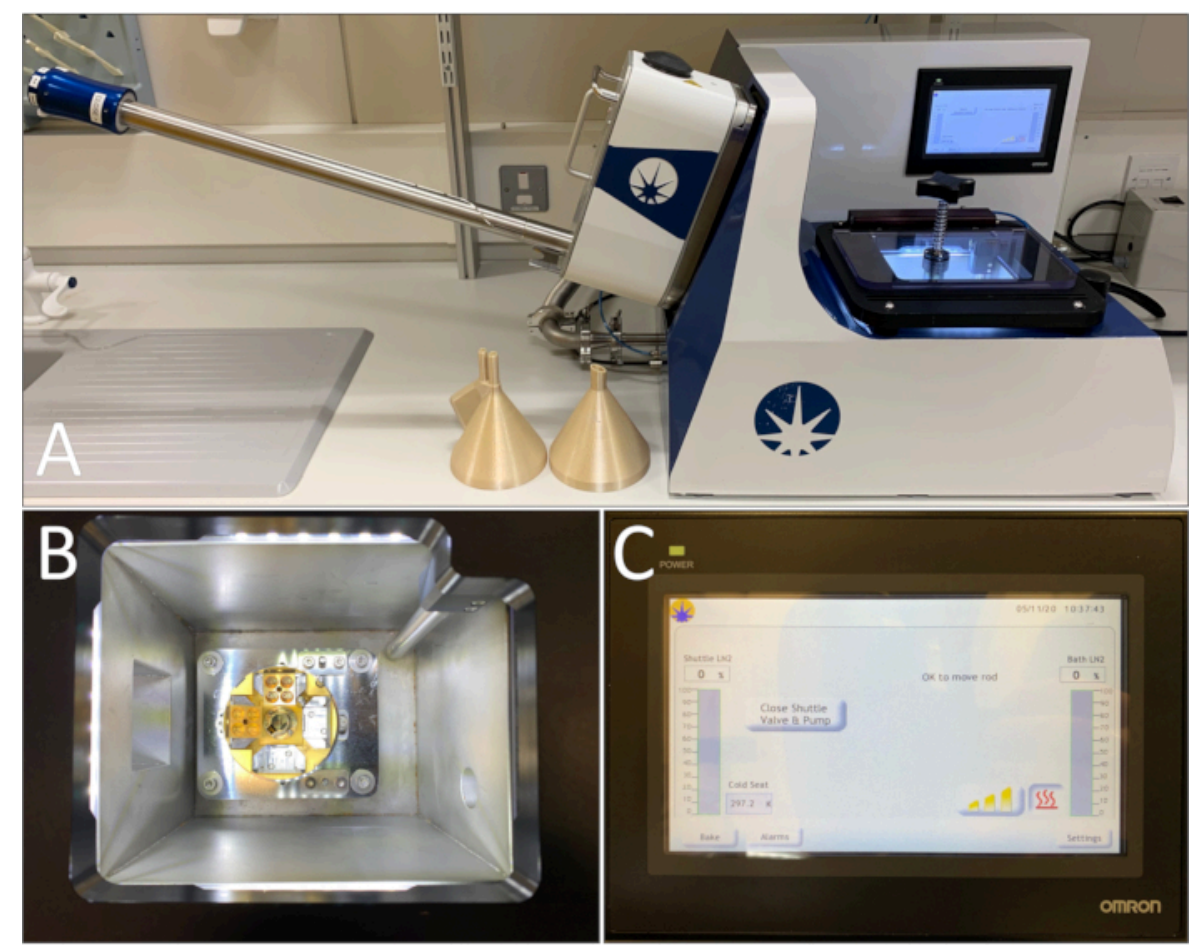

Figure 4: Cryogenic Transfer System. (A) CTS Sample station with shuttle attached and the funnels used for filling. (B) A block puck with two transfer blocks positioned inside the CTS. (C) CTS control software touchscreen. Abbreviation: CTS = Cryogenic Transfer System. Please click here to view a larger version of this figure. 


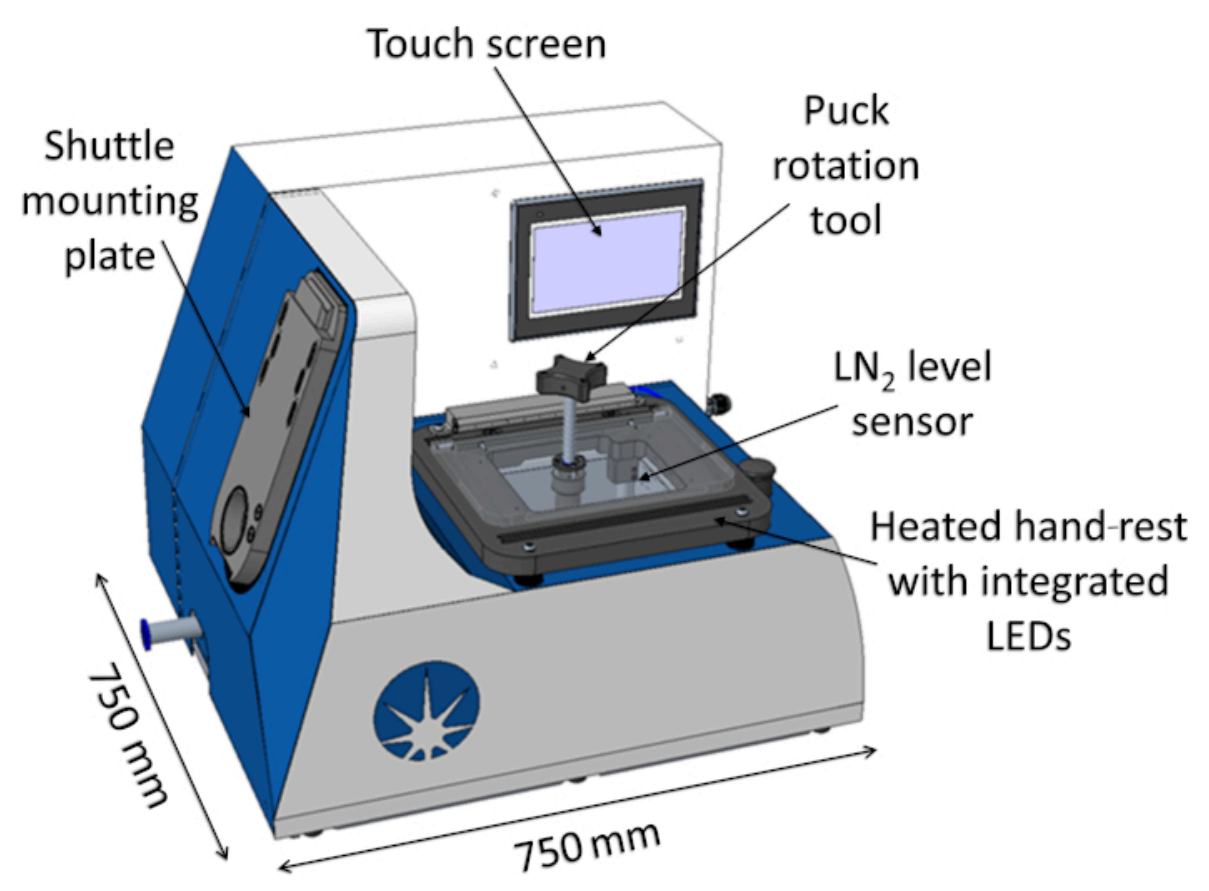

Figure 5: Cryogenic Transfer System Sample Station. Abbreviations: LEDs = light-emitting diodes; LN2 = liquid nitrogen. Please click here to view a larger version of this figure. 


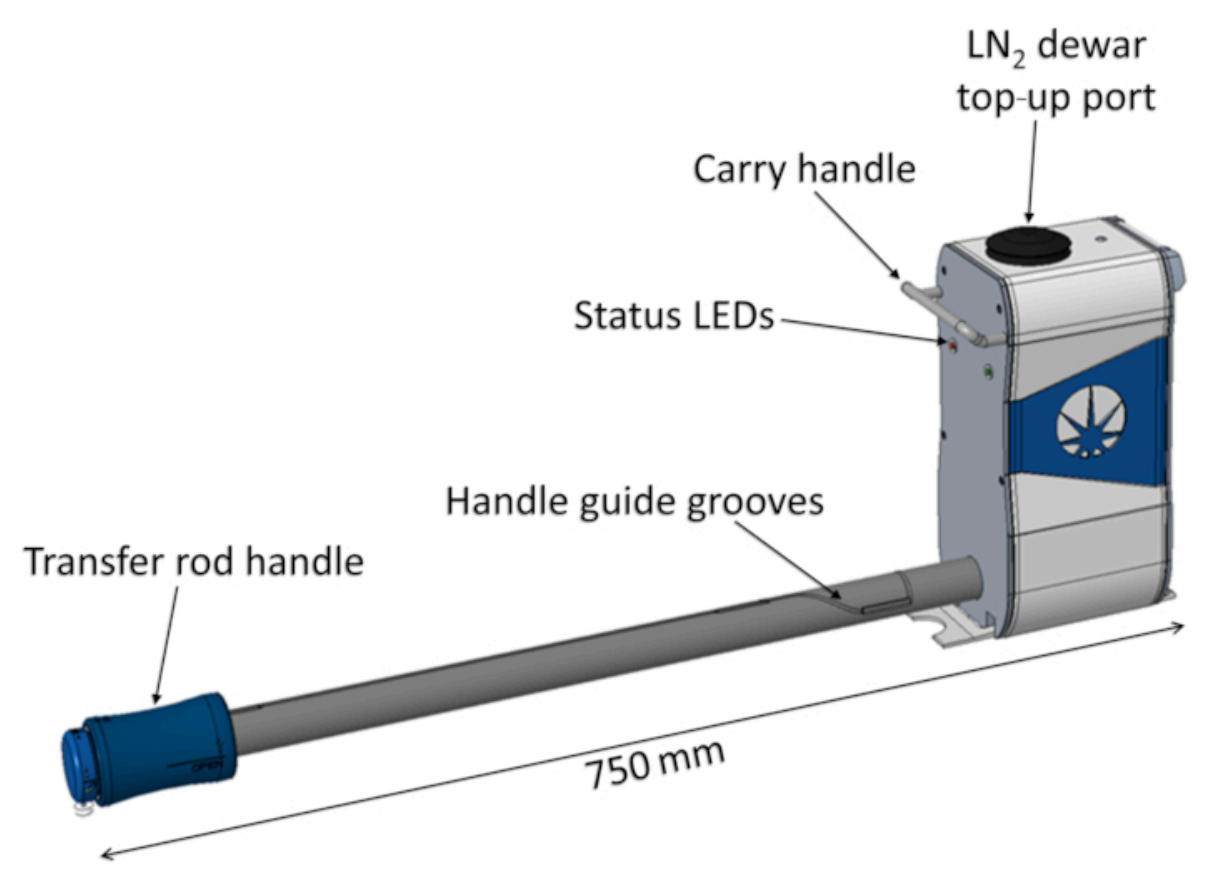

Figure 6: Cryogenic Transfer System Shuttle. Abbreviations: LEDs = light-emitting diodes; LN2 = liquid nitrogen. Please click here to view a larger version of this figure. 
Figure 7: Diffraction images. Left, a diffraction image from the dataset collected on the thaumatin crystal. Right, a diffraction spot surrounded by low-count background pixels. Please click here to view a larger version of this figure. 


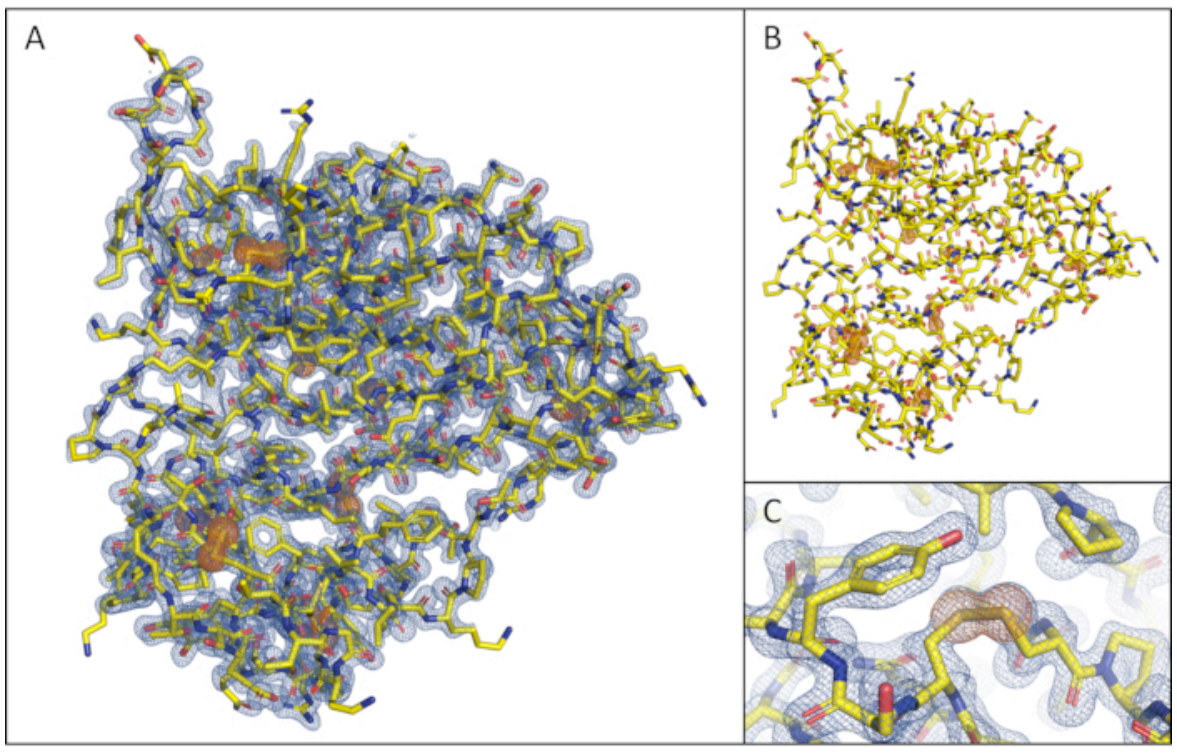

Figure 8: Structure solution of Thaumatin with automatic pipeline CRANK2 (default settings, no subsequent

refinement). (A) Overview of thaumatin with $2 \mathrm{~F}_{\mathrm{O}}-\mathrm{F}_{\mathrm{C}}$ map at $1.6 \sigma$ (blue) and phased anomalous difference Fourier map at $5 \sigma$ calculated in ANODE (green). (B) Overview of thaumatin showing only the phased anomalous difference Fourier map at $5 \sigma$. (C) Close-up view of a disulfide bridge present in thaumatin with $2 \mathrm{Fo}-\mathrm{Fc}$ map at $1.6 \sigma$ (blue) and phased anomalous difference Fourier map at $5 \sigma$. Please click here to view a larger version of this figure. 


\begin{tabular}{|c|c|}
\hline Name & Thaumatin \\
\hline Data collection wavelength $(\AA)$ (energy $(\mathrm{eV}))$ & $2.7552(4500)$ \\
\hline Number of images $x$ wedge size $\left({ }^{\circ}\right)$ & $3600 \times 0.1$ \\
\hline Spacegroup & $P 41212$ \\
\hline \multicolumn{2}{|l|}{ Unit cell constants } \\
\hline$(a=b, c)(A)$ & $57.8,150.2$ \\
\hline$(\alpha=\beta=y)\left({ }^{\circ}\right)$ & 90 \\
\hline Resolution $(\AA)$ & $150.22-1.80(1.84-1.80)$ \\
\hline Completeness & $96.3(81.1)$ \\
\hline ISa & 36.48 \\
\hline$R_{\text {meas }}$ & $0.042(0.118)$ \\
\hline$R_{\text {pim }}$ & $0.01(0.049)$ \\
\hline$C C_{1 / 2}$ & $1(0.989)$ \\
\hline$I / \sigma(I)$ & $57.9(14.7)$ \\
\hline Multiplicity & $15.0(5.4)$ \\
\hline Mid-slope & 2.677 \\
\hline
\end{tabular}

Table 1: Data collection and processing statistics for Thaumatin at 2.755 A wavelength at beamline I23, DLS.

For resolution, completeness, $R_{\text {merge }}, R_{\text {meas }}, R_{\text {pim }}, C_{1 / 2}, I / \sigma(I)$, and multiplicity, high-resolution shells are shown in parentheses. Abbreviation: DLS = Diamond Light Source. 


\begin{tabular}{|c|c|}
\hline Nearest atom & Peak height (sigma) \\
\hline CYS9 & 25.83 \\
\hline CYS56 & 25.03 \\
\hline MET112 & 24.54 \\
\hline CYS149 & 24.37 \\
\hline CYS126 & 24.21 \\
\hline CYS145 & 24.2 \\
\hline CYS134 & 23.6 \\
\hline CYS177 & 23.48 \\
\hline CYS204 & 23.43 \\
\hline CYS66 & 23.17 \\
\hline CYS164 & 22.54 \\
\hline CYS193 & 22.15 \\
\hline CYS158 & 21.51 \\
\hline CYS77 & 21.21 \\
\hline CYS121 & 20.8 \\
\hline CYS71 & 19.17 \\
\hline CYS159_1 & 12.27 \\
\hline CYS159_2 & 8.34 \\
\hline
\end{tabular}

Table 2: Anomalous difference Fourier map peak heights as calculated by ANODE using the phased and automatically built model from CRANK2.

\section{Discussion}

The current protocol has been developed to comply with the sample preparation requirements for in-vacuum longwavelength $\mathrm{MX}$ experiments on beamline 123 . It has been in use on the beamline for the past year and has contributed to the successful completion of multiple projects. As indicated by the results presented here, the protocol enables a safe and reliable transfer of samples to the vacuum end-station while preserving their diffraction quality. It is an important aspect for the beamline operation and will be accompanied by inperson user training by beamline staff. Some of the steps are worth being highlighted as critical to the successful and safe completion of the procedure: the transfer of samples from combipuck bases to sample blocks requires accuracy 
and attention to avoid damaging samples (see step 2.1.4); monitoring of the liquid nitrogen level at all stages is important to prevent samples being exposed to air or being in close contact to parts that are not properly cooled (2.1.3 and 2.2.2); waiting until the Close sequence (2.2.14) is completely finished, before removing the shuttle from the endstation (2.2.15), to avoid degradation of the endstation vacuum.

The conception of the protocol was initiated together with an engineering effort aimed at developing purpose-built equipment for the transfer of protein crystals to the vacuum environment. The final products of this project were the CTS and the associated sample handling tools described above. The CTS is a significant improvement on its predecessor, the Leica EM VCT100 ${ }^{14}$, and removes multiple limitations, such as the lack of sample shielding and vacuum environment during transfer, ice build-up inside the liquid nitrogen bath, and the absence of an intuitive user interface and safety features. Additional features of the CTS that improve the user experience are temperature and liquid nitrogen level monitoring inside the shuttle and sample station, a larger capacity bath accommodating four blocks simultaneously, rather than one, and a self-guided mechanism for the shuttle operation. The CTS is fully integrated into the beamline control system with a user-friendly touchscreen interface and enhanced vacuum and mechanical safety when interfacing with the endstation.

Beamline I23 is the first long-wavelength MX synchrotron instrument of its kind and, as such, introducing protein crystals to a high-vacuum environment and storing them at cryogenic temperatures, has required considerable efforts. Improvements to the sample preparation tools and protocol, as well as efforts to streamline processes, are on-going. As part of user support, beamline staff are always available to assist with troubleshooting. An example of one such scenario would be issues that compromise the integrity of the vacuum system, leading to difficulties in attaching or removing the shuttle to/from either the CTS or endstation airlock. Different levels of tests are performed on a weekly and daily basis, and user training will cover additional checks to avoid potential failures, like visual inspection of the $O$ rings on the interfaces the shuttle attaches to. While the vacuum environment opens the opportunity to perform diffraction experiments in a wavelength range not accessible at other beamlines, the additional transfer step reduces the overall sample throughput.

The manual transfer with only four samples per transfer block and up to five blocks inside the vacuum vessel limits the total capacity to 20 samples. Hence, for projects with a large sample to sample variability, samples should be pre-screened at the Diamond high-throughput beamlines, and then only the most promising samples should be transferred for the subsequent optimized long-wavelength experiment. While the sample holders and the transfer blocks are unchanged from their initial introduction a few years ago, the handling tools presented here are all new developments. The 123 dedicated sample holders are immutable due to their role in the cooling concept for the beamline. As such, the design of the sample handling tools aimed to create a link between this new type of holder and standard commercially available tools that the MX user community had adopted for a long time, such as combipucks, crystal harvesting wands, and the dry shipper transport system. Their design involved significant consultation with the user community and required several iterations to complete. The equipment, tools, and protocol presented here represent a simple and robust system for the transfer of user samples for experiments at beamline I23 at Diamond Light Source. This instrument for in-vacuum 
long-wavelength macromolecular crystallography opens new opportunities for structural biology.

\section{Acknowledgments}

We would like to thank Adam Taylor, Adam Prescott, Ken Jones, Arvinder Palaha, and Kevin Wilkinson for their support in the development of the Cryogenic Sample Transfer System (CTS). This work was funded by iNEXT-Discovery (Grant 871037) funded by Horizon 2020 program of the European Commission.

\section{References}

1. Green, D. W.; Ingram, V. M., Perutz, M. F. The structure of haemoglobin - IV. Sign determination by the isomorphous replacement method. Proceedings of the Royal Society of London. Series A. Mathematical and Physical Sciences. 225 (1162), 287-230 (1954).

2. Hendrickson, W. A. Anomalous diffraction in crystallographic phase evaluation. Quarterly Reviews of Biophysics. 47 (1), 49-93 (2014).

3. Pike, A. C., Garman, E. F., Krojer, T., von Delft, F., Carpenter, E. P. An overview of heavy-atom derivatization of protein crystals. Acta Crystallographica Section D: Structural Biology. 72 (3), 303-318 (2016).

4. Hendrickson, W. A., Horton, J. R., LeMaster, D. M. Selenomethionyl proteins produced for analysis by multiwavelength anomalous diffraction (MAD): A vehicle for direct determination of three-dimensional structure. The EMBO Journal. 9 (5), 1665-1672 (1990).

5. Liu, Q., Hendrickson, W. A. Contemporary use of anomalous diffraction in biomolecular structure analysis. In: Wlodawer A., Dauter Z., Jaskolski M. (eds) Protein
Crystallography. Methods in Molecular Biology,. 1607. Humana Press, New York, NY. 377-399 (2017).

6. Rose, J. P., Wang, B. C., Weiss, M. S. Native SAD is maturing.IUCrJ. 2 (4), 431-440 (2015).

7. Rozov, A. et al. Importance of potassium ions for ribosome structure and function revealed by longwavelength X-ray diffraction. Nature Communications. 10 (1), 2519 (2019).

8. Rocchio, S. et al. Identifying dynamic, partially occupied residues using anomalous scattering. Acta Crystallographica Section D: Structural Biology. 75 (12), 1084-1095 (2019).

9. Langan, P. S. et al. Anomalous X-ray diffraction studies of ion transport in $\mathrm{K}^{+}$channels. Nature Communications. 9 (1), 4540 (2018).

10. Lolicato, $M$. et al. $\mathrm{K}_{2 \mathrm{p}}$ channel $\mathrm{C}$-type gating involves asymmetric selectivity filter order-disorder transitions. Science Advances. 6 (44), eabc9174 (2020).

11. Thorn, A., Sheldrick, G. M. ANODE: anomalous and heavy-atom density calculation. Journal of Applied Crystallography. 44 (6), 1285-1287 (2011).

12. Handing, K. B., Niedzialkowska, E., Shabalin, I. G., Kuhn, M. L., Zheng, H., Minor, W. Characterizing metalbinding sites in proteins with X-ray crystallography. Nature Protocols. 13 (5), 1062-1090 (2018).

13. Jungnickel, K. E. J., Parker, J. L., Newstead, S. Structural basis for amino acid transport by the CAT family of SLC7 transporters. Nature Communications. 9 (1), 550 (2018).

14. Wagner, A., Duman, R., Henderson, K., Mykhaylyk, V. In-vacuum long-wavelength macromolecular crystallography. Acta Crystallographica Section D: Structural Biology. 72 (3), 430-439 (2016). 
15. Wernecke, J., Gollwitzer, C., Müller, P., Krumrey, M. Characterization of an in-vacuum PILATUS 1M detector. Journal of Synchrotron Radiation. 21 (3), 529-536 (2014).

16. Kabsch, W. XDS. Acta Crystallographica Section D: Biological Crystallography. 66 (2), 125-132 (2010).

17. Winter, G. et al. DIALS: Implementation and evaluation of a new integration package. Acta Crystallographica Section D: Structural Biology. 74 (2), 85-97 (2018).

18. Brockhauser, S., Di Michiel, M., Mcgeehan, J. E., Mccarthy, A. A., Ravelli, R. B. G. X-ray tomographic reconstruction of macromolecular samples. Journal of Applied Crystallography. 41 (6), 1057-1066 (2008).

19. Kitano, $H$. et al. Processing of membrane protein crystal using ultraviolet laser irradiation. Journal of Bioscience and Bioengineering. 100 (1), 50-53 (2005).

20. Mykhaylyk, V., Wagner, A. Towards long-wavelength protein crystallography: Keeping a protein crystal frozen in vacuum. Journal of Physics: Conference Series. 425 (1), 012010 (2013).

21. Snell, G. et al. Automated sample mounting and alignment system for biological crystallography at a synchrotron source. Structure. 12 (4), 537-545 (2004).

22. The universal container project. https:// smb.slac.stanford.edu/robosync/Universal_Puck/ (2006).

23. Teng, T. Y. Mounting of crystals for macromolecular crystallography in a freestanding thin-film. Journal of Applied Crystallography. 23, 387-391 (1990).

24. Esposito, D. et al. Structural basis for the glycosyltransferase activity of the salmonella effector SseK3. Journal of Biological Chemistry. 293 (14), 5064-5078 (2018).
25. O'Donnell, J. P. et al. The architecture of EMC reveals a path for membrane protein insertion. eLife. 9, e57887 (2020).

26. Mishra, A. K. et al. Structure and characterization of crimean-congo hemorrhagic fever virus GP38. Journal of Virology. 94 (8), e02005-19 (2020).

27. Rudolf, A. F. et al. The morphogen sonic hedgehog inhibits its receptor patched by a pincer grasp mechanism. Nature Chemical Biology. 15 (10), 975-982 (2019).

28. El Omari, K. et al. Pushing the limits of sulfur sad phasing: De novo structure solution of the n-terminal domain of the ectodomain of hcv e1. Acta Crystallographica Section D: Structural Biology. 70 (8), 2197-2203 (2014).

29. Winter, G. XIA2: an expert system for macromolecular crystallography data reduction. Journal of Applied Crystallography. 43 (1), 186-190 (2010).

30. Cowtan, K. The Buccaneer software for automated model building. 1. Tracing protein chains. Acta Crystallographica Section D: Structural Biology. 62 (9), 1002-1011 (2006).

31. Skubak, P., Pannu, N. S. Automatic protein structure solution from weak X-ray data. Nature Communications. 4 (1), 2777 (2013). 